\title{
Measurements of charmless B decays related to $\alpha$ at BABAR
}

\author{
Vincenzo LOMBARDO* (for the BABAR Collaboration) \\ INFN Milano (Italy) \\ E-mail: vincenzo.lombardo@mi.infn.it
}

\begin{abstract}
We report recent measurements of the CKM angle $\alpha$ using data collected by the BABAR detector at the PEP-II asymmetric-energy $e^{+} e^{-}$collider at the SLAC National Accelerator Laboratory. In addition to improved constraints on $\alpha$ from the decays $B^{ \pm} \rightarrow \rho^{ \pm} \rho^{0}$, we also present preliminary results of neutral and charged $B$ meson decays to $K_{1}(1270) \pi$ and $K_{1}(1400) \pi$ and its impact on the estimate for the CKM angle $\alpha$ based on time-dependent analysis of CP-violating asymmetries in $B^{0} \rightarrow a_{1}(1260)^{ \pm} \pi^{\mp}$. Moreover we report the first observation of the decay $B \rightarrow a_{1}(1260)^{ \pm} a_{1}(1260)^{\mp}$; this mode can be used, in principle, to provide an independent measurement of $\alpha$.
\end{abstract}

European Physical Society Europhysics Conference on High Energy Physics

July 16-22, 2009

Krakow, Poland

\footnotetext{
* Speaker.
} 


\section{Introduction}

The measurements of the angles $\alpha, \beta$ and $\gamma$ of the Unitarity Triangle (UT) at the B-factories are providing precision tests of the description of CP violation in the Standard Model (SM). This description is provided by the Cabibbo-Kobayashi-Maskawa (CKM) quark-mixing matrix [1, 2]. I am summarizing here recent experimental results on the Unitarity Triangle angle $\alpha$ obtained from B-meson decays with the BABAR experiment at the SLAC National Accelerator Laboratory. The BABAR detector and PEP-II accelerator are described elsewhere [3]. The decays of neutral $B$ mesons to the final states $h^{+} h^{\prime}$, where $h^{+}, h^{\prime}-\pi, \rho, a_{1}$ are sensitive to the CKM angle $\alpha$ in the interference between decay and mixing [ [†. The presence of gluonic loop ("penguin") contributions with a different weak phase to the tree contribution shifts the measured angle from the UT angle $\alpha$ to an effective parameter $\alpha_{e f f}$, where the shift is defined as $\delta \alpha=\alpha-\alpha_{e f f}$. Either isospin symmetry [5, 6] or broken SU(3) flavour symmetry [7] can be employed to disentangle $\alpha$ from $\alpha_{e f f}$.

\section{Measurement of $B$ decays to $\rho^{ \pm} \rho^{0}$}

One of the most favourable methods to determine $\alpha$ is through an isospin analysis of the $B \rightarrow \rho \rho$ system [8, 9]. Here we present updated BaBar results for $B^{+} \rightarrow \rho^{+} \rho^{0}$ channel, with $\rho^{+} \rightarrow \pi^{+} \pi^{0}$ and $\rho^{0} \rightarrow \pi^{+} \pi^{-}$, leading to an improved determination of $\alpha$ [10]. The analysis is based on $(465 \pm$ $5) \times 10^{6} B \bar{B}$ events collected on the $Y(4 S)$ resonance. Compared to the previous study [11], the analysis incorporates higher signal efficiency and background rejection, twice as much as data, and an improved procedures to reconstruct charged particles and to account for correlations in the backgrounds. The measured branching fraction $\mathscr{B}\left(B^{+} \rightarrow \rho^{+} \rho^{0}\right)=(23.6 \pm 1.4 \pm 1.4) \times 10^{-6}$ is larger than Ref. [11], primarily because of the improved method used here to account for correlations in the backgrounds. The measured longitudinal polarization fraction is $f_{L}=0.950 \pm 0.015 \pm 0.006$. An isospin analysis of $B \rightarrow \rho \rho$ has been performed by minimizing a $\chi^{2}$ that included the measured quantities expressed as the lengths of the sides of the $B$ and $\bar{B}$ isospin triangles [5]. The $B^{+} \rightarrow \rho^{+} \rho^{0}$ branching fraction and $f_{L}$ results presented here have been used, with the branching fractions, polarizations and CP-violating parameters in $B^{0} \rightarrow \rho^{+} \rho^{-}\left[12\right.$ and $B^{0} \rightarrow \rho^{0} \rho^{0}$ decays reported in Ref. [13]. We assume the uncertainties to be Gaussian-distributed and neglect potential isospin $I=1$ and electroweak-loop amplitudes which are expected to be small. The CKM phase angle $\alpha$ and its correction $\Delta \alpha$ are found to be $\alpha=\left(92.4_{-6.5}^{+6.0}\right)^{\circ}$ and $1.8^{\circ}<\Delta \alpha<6.7^{\circ}$, respectively at $68 \%$ C.L., significant improvement compared to $\alpha=\left(82.6_{-6.3}^{+32.6}\right)^{\circ}$ and $|\Delta \alpha|<15.7^{\circ}$ obtained with the same $\rho^{+} \rho^{-}$and $\rho^{0} \rho^{0}$ measurements but the previous $B^{+} \rightarrow \rho^{+} \rho^{0}$ results. The improvement is primarily due to the increase in $\mathscr{B}\left(B^{+} \rightarrow \rho^{+} \rho^{0}\right)$ compared to our previous result. $\mathscr{B}\left(B^{+} \rightarrow \rho^{+} \rho^{0}\right)$ determines the length of the common base of isospin triangles for $B$ and $\bar{B}$ decays. The increase in the base length flattens both triangles, making the four possible solutions nearly degenerate.

\section{Measurement of B decays to $K_{1}(1270) \pi$ and $K_{1}(1400) \pi$}

BaBar has recently reported the measurement of branching fractions of neutral and charged $B$ meson decays to $K_{1}(1270) \pi$ and $K_{1}(1400) \pi$, obtained from a data sample of 454 million $\Upsilon(4 S) \rightarrow B \bar{B}$ 
events [14]. The signal is modelled with a $K$-matrix formalism, which accounts for the effects of interference between the $K_{1}(1270)$ and $K_{1}(1400)$ mesons. Including systematic and model uncertainties, BaBar measures $\mathscr{B}\left(B^{0} \rightarrow K_{1}(1270)^{+} \pi^{-}+K_{1}(1400)^{+} \pi^{-}\right)=3.1_{-0.7}^{+0.8} \times 10^{-5}$ and $\mathscr{B}\left(B^{+} \rightarrow\right.$ $\left.K_{1}(1270)^{0} \pi^{+}+K_{1}(1400)^{0} \pi^{+}\right)=2.9_{-1.7}^{+2.9} \times 10^{-5}\left(<8.2 \times 10^{-5}\right.$ at $90 \%$ probability $)$. A combined signal for the decays $B^{0} \rightarrow K_{1}(1270)^{+} \pi^{-}$and $B^{0} \rightarrow K_{1}(1400)^{+} \pi^{-}$is observed with a significance of $7.5 \sigma$, and the following branching fractions are derived for neutral $B$ meson decays: $\mathscr{B}\left(B^{0} \rightarrow K_{1}(1270)^{+} \pi^{-}\right) \in[0.6,2.5] \times 10^{-5}, \quad \mathscr{B}\left(B^{0} \rightarrow K_{1}(1400)^{+} \pi^{-}\right) \in[0.8,2.4] \times 10^{-5}$, and $\mathscr{B}\left(B^{0} \rightarrow K_{1 A}^{+} \pi^{-}\right) \in[0.4,2.3] \times 10^{-5}$, where the $K_{1 A}$ meson is a nearly equal admixture of the $K_{1}(1270)$ and $K_{1}(1400)$ resonances [8] and where the two-sided intervals are evaluated at $68 \%$ probability. A significance of $3.2 \sigma$ is obtained for $B^{+} \rightarrow K_{1}(1270)^{0} \pi^{+}+K_{1}(1400)^{0} \pi^{+}$, and the following two-sided intervals at $68 \%$ probability and upper limits at $90 \%$ probability are derived: $\mathscr{B}\left(B^{+} \rightarrow K_{1}(1270)^{0} \pi^{+}\right) \in[0.0,2.1] \times 10^{-5}\left(<4.0 \times 10^{-5}\right), \quad \mathscr{B}\left(B^{+} \rightarrow K_{1}(1400)^{0} \pi^{+}\right) \in$ $[0.0,2.5) \times 10^{-5}\left(<3.9 \times 10^{-5}\right)$, and $\mathscr{B}\left(B^{+} \rightarrow K_{1 A}^{0} \pi^{+}\right) \in[0.0,2.1] \times 10^{-5}\left(<3.6 \times 10^{-5}\right)$. Moreover BaBar has combined these branching fractions with existing experimental information to derive an independent estimate for the CKM angle $\alpha$, based on the time-dependent analysis of $C P$-violating asymmetries in $B^{0} \rightarrow a_{1}(1260)^{ \pm} \pi^{\mp}$ [15]. The $\Delta S=1$ decays presented here are particularly sensitive to the presence of penguin amplitudes because their CKM couplings are larger than the corresponding $\Delta S=0$ penguin amplitudes. Thus measurements of the decay rates of the $\Delta S=1$ transitions involving the same $\mathrm{SU}(3)$ flavour multiplet as $a_{1}(1260)$ provide constraints on $\Delta \alpha=\alpha_{\text {eff }}-\alpha[16]$. Similar SU(3)-based approaches have been proposed for the extraction of $\alpha$ in the $\pi^{+} \pi^{-}$[17], $\rho^{ \pm} \pi^{\mp}$ [7], and $\rho^{+} \rho^{-}$channels [18, 12]. Babar derives bounds on the model uncertainty $|\Delta \alpha|$ on the weak phase $\alpha_{\text {eff }}$ extracted in $B^{0} \rightarrow a_{1}(1260)^{ \pm} \pi^{\mp}$ decays using previously measured branching fractions of $B^{0} \rightarrow a_{1}(1260)^{ \pm} \pi^{\mp}, B^{0} \rightarrow a_{1}(1260)^{-} K^{+}$and $B^{+} \rightarrow a_{1}(1260)^{+} K^{0}$ decays [19] and the $C P$-violation asymmetries [15] as input to the method of Ref. [16] and obtains $\left|\alpha-\alpha_{\text {eff }}\right|<11^{\circ}\left(13^{\circ}\right)$ at $68 \%(90 \%)$ probability. The determination of $\alpha_{\text {eff }}$ presents an eightfold ambiguity in the range $\left[0^{\circ}, 180^{\circ}\right]$. The eight solutions are $\alpha_{\mathrm{eff}}=(11 \pm 7)^{\circ}, \alpha_{\mathrm{eff}}=(41 \pm 7)^{\circ}$, $\alpha_{\text {eff }}=(49 \pm 7)^{\circ}, \alpha_{\text {eff }}=(79 \pm 7)^{\circ}, \alpha_{\text {eff }}=(101 \pm 7)^{\circ}, \alpha_{\text {eff }}=(131 \pm 7)^{\circ}, \alpha_{\text {eff }}=(139 \pm 7)^{\circ}, \alpha_{\text {eff }}=$ $(169 \pm 7)^{\circ}$ [15]. Assuming that the relative strong phase between the relevant tree amplitudes is negligible [16] it is possible to reduce this ambiguity to a twofold ambiguity in the range $\left[0^{\circ}, 180^{\circ}\right]$ : $\alpha_{\text {eff }}=(11 \pm 7)^{\circ}, \alpha_{\text {eff }}=(79 \pm 7)^{\circ}$. BaBar combines the solution near $90^{\circ}, \alpha_{\text {eff }}=(79 \pm 7)^{\circ}$, with the bounds on $\left|\alpha_{\text {eff }}-\alpha\right|$ and estimates the weak phase $\alpha=(79 \pm 7 \pm 11)^{\circ}$. This solution is consistent with the current average value of $\alpha$, based on the analysis of $B \rightarrow \pi \pi, B \rightarrow \rho \rho$, and $B \rightarrow \rho \pi$ decays [8, 9].

\section{Measurement of B decays to $a_{1}(1260)^{ \pm} a_{1}(1260)^{\mp}$}

Charmless $B$ decays to final states involving two axial-vector mesons (AA) have received considerable theoretical attention in the last few years [20, 21]. Using QCD factorization, the branching fractions of several $B \rightarrow A A$ decay modes have been calculated. Predictions for the branching fraction of the $B^{0} \rightarrow a_{1}(1260)^{ \pm} a_{1}(1260)^{\mp}$ [22] decay mode vary between $37.4 \times 10^{-6}$ [20] and $6.4 \times 10^{-6}[21]$. Branching fractions at this level should be observable with the BaBar data sample, which can be used to discriminate between the predictions. The predicted value of the longitudinal polarization fraction $f_{L}$ is 0.64 [20]. The only available experimental information on this $B$ decay 
mode is the branching fraction upper limit (UL) of $2.8 \times 10^{-3}$ at $90 \%$ confidence level (CL) measured by CLEO [23]. The measured value $f_{L} \sim 0.5$ in penguin-dominated $B \rightarrow \phi K^{*}$ decays [24] is in contrast with naive $\mathrm{SM}$ calculations predicting a dominant longitudinal polarization $\left(f_{L} \sim 1\right)$ in $B$ decays to vector-vector (VV) final states. The naive SM expectation is confirmed in the treedominated $B \rightarrow \rho \rho$ [12, 25, 10] and $B^{+} \rightarrow \omega \rho^{+}$[26] decays. A value of $f_{L} \sim 1$ is found in vectortensor $B \rightarrow \phi K_{2}^{*}(1430)$ decays [27], while $f_{L} \sim 0.5$ is found in $B \rightarrow \omega K_{2}^{*}(1430)$ decays [26] (see Ref [28] for further discussion). The small value of $f_{L}$ observed in $B \rightarrow \phi K^{*}$ decays has stimulated theoretical effort, such as the introduction of non-factorizable terms and penguin-annihilation amplitudes [29]. Other explanations invoke new physics [30]. Measurement of $f_{L}$ in $a_{1} a_{1}$ [22] decays will provide additional information. The analysis uses an integrated luminosity of $423.0 \mathrm{fb}^{-1}$, corresponding to $(465 \pm 5) \times 10^{6} B \bar{B}$ pairs, recorded at the $\Upsilon(4 S)$ resonance [31]. BaBar has measured the branching fraction: $\mathscr{B}\left(B^{0} \rightarrow a_{1}^{+} a_{1}^{-}\right) \times\left[\mathscr{B}\left(a_{1}^{+} \rightarrow(3 \pi)^{+}\right)\right]^{2}=11.8 \pm 2.6 \pm 1.6 \times 10^{-6}$ and the fraction of longitudinal polarization $f_{L}=0.31 \pm 0.22 \pm 0.10$. Assuming that $\mathscr{B}\left(a_{1}^{+} \rightarrow \pi^{-} \pi^{+} \pi^{+}\right)$is equal to $\mathscr{B}\left(a_{1}^{+} \rightarrow \pi^{+} \pi^{0} \pi^{0}\right)$, and that $\mathscr{B}\left(a_{1}^{+} \rightarrow(3 \pi)^{+}\right)$is equal to $100 \%$ [ 8 ] the branching fraction is $\mathscr{B}\left(B^{0} \rightarrow a_{1}^{+} a_{1}^{-}\right)=(47.3 \pm 10.5 \pm 6.3) \times 10^{-6}$. The decay mode is seen with a significance of $5.0 \sigma$, which includes systematic uncertainties. The measured branching fraction and longitudinal polarization are in general agreement with the theoretical expectations in [20]. In principle an independent measurement of $\alpha$ of the UT can be extracted from the time-dependent analysis of $C P$-violating asymmetries of $B^{0} \rightarrow a_{1}(1260)^{ \pm} a_{1}(1260)^{\mp}$ but the available statistics is indeed too low to perform such an analysis. Potentially a new generation of Super Flavour Factories [33, 32] will be able to achieve this result.

\section{Summary}

In summary significant progress in the measurement of $\alpha$ has been made over the last decade by BaBar. In $B \rightarrow \rho \rho$ decays recent Babar results have substantially improved the precision of the measurement and at the same time a new measurement of $\alpha$ has been extracted from $B \rightarrow$ $a_{1}(1260) \pi$ decays. All these results are in good agreement with the predictions obtained by SMbased fits [阿.

\section{References}

[1] N. Cabibbo Phys. Rev. lett. 10, 531 (1963); M. Kobayashi and T. Maskawa, Prog. Theor. Phys. 49, 652 (1973).

[2] L. Wolfenstein, Phys. Rev. Lett. 51, 1945 (1983).

[3] B. Aubert et al. [BABAR Collaboration], Nucl. Instrum. Meth. A 479, 1 (2002).

[4] For the $a_{1}(1260)$ meson we use the short notation $a_{1}$.

[5] M. Gronau and D. London, Phys. Rev. Lett. 65, 3381 (1990); H. J. Lipkin et al., Phys. Rev. D 44, 1454 (1991); M. Gronau, Phys. Lett. B 265, 389 (1991).

[6] Y. Grossman and H. R. Quinn Phys. Rev. D 58, 017504 (1998); J. Charles,Phys. Rev. D 59, 054007 (1999); M. Gronau et al., Phys. Lett. B 514, 315 (2001).

[7] M. Gronau and J. Zupan, Phys. Rev. D 70, 074031 (2004). 
[8] C. Amsler et al.(Particle Data Group), Phys. Lett. B, 667, 1 (2008).

[9] J. Charles et al.(CKMfitter Group), Eur. Phys. J. C. 41, 1 (2005); M. Bona et al.(UTfit Collaboration), J. High Energy Phys. 03 (2006) 080.

[10] B. Aubert et al.(BaBar Collaboration) Phys. Rev. Lett. 102, 141802 (2009).

[11] B. Aubert et al.(BaBar Collaboration) Phys. Rev. Lett. 97, 261801 (2006).

[12] B. Aubert et al.(BaBar Collaboration) Phys. Rev. D 76, 052007 (2007).

[13] B. Aubert et al.(BaBar Collaboration) Phys. Rev. D 78, 071104 (2008).

[14] BaBar Collaboration, B. Aubert et al., arXiv:0909.2171 [hep-ex]

[15] BaBar Collaboration, B. Aubert et al., Phys. Rev. Lett. 98, 181803 (2007).

[16] M. Gronau and J. Zupan, Phys. Rev. D 73, 057502 (2006);

[17] M. Gronau and J. L. Rosner, Phys. Lett. B 595, 339 (2004).

[18] M. Beneke, M. Gronau, J. Rohrer, and M. Spranger, Phys. Lett. B 638, 68 (2006).

[19] BaBar Collaboration, B. Aubert et al., Phys. Rev. Lett. 97, 051802 (2006); Phys. Rev. Lett. 100, 051803 (2008).

[20] H.-Y. Cheng and K.-C. Yang, Phys. Rev. D 78, 094001 (2008).

[21] G. Calderon et al., Phys. Rev. D 76, 094019 (2007).

[22] $a_{1}$ will be used to indicate the $a_{1}(1260)$ meson.

[23] CLEO Collaboration, D. Bortoletto et al., Phys. Rev. Lett. 62, 2436 (1989).

[24] BaBar Collaboration, B. Aubert et al., Phys. Rev. D 78, 092008 (2008); Belle Collaboration, K. F. Chen et al., Phys. Rev. Lett. 91, 201801 (2003).

[25] Belle Collaboration, A. Somov et al., Phys. Rev. Lett. 96, 171801 (2006); Belle Collaboration, J. Zhang et al., Phys. Rev. Lett. 91, 221801 (2003);

[26] BaBar Collaboration, B. Aubert et al., Phys. Rev. D 79, 052005.

[27] BaBar Collaboration, B. Aubert et al., Phys. Rev. Lett. 98, 051801 (2007).

[28] A. V. Gritsan and J. G. Smith, "Polarization in B Decays" review in [8], Phys. Lett. B 667, 910 (2008).

[29] A. L. Kagan, Phys. Lett. B 601, 151 (2004); C. W. Bauer et al., Phys. Rev. D 70, 054015 (2004); P. Colangelo, F. De Fazio, and T. N. Pham, Phys. Lett. B 597, 291 (2004); M. Ladisa et al., Phys. Rev. D 70, 114025 (2004); H. Y. Cheng, C. K. Chua, and A. Soni, Phys. Rev. D 71, 014030 (2005); H. N. Li and S. Mishima, Phys. Rev. D 71, 054025 (2005); C. H. Chen et al., Phys. Rev. D 72, 054011 (2005); M. Beneke et al., Nucl. Phys. B 774, 64 (2007).

[30] A. K. Giri and R. Mohanta, Phys. Rev. D 69, 014008 (2004); E. Alvarez et al., Phys. Rev. D 70, 115014 (2004); P. K. Das and K. C. Yang, Phys. Rev. D 71, 094002 (2005); C. H. Chen and C. Q. Geng, Phys. Rev. D 71, 115004 (2005); Y. D. Yang, R. M. Wang, and G. R. Lu, Phys. Rev. D 72, 015009 (2005); C. S. Hunger et al., Phys. Rev. D 73, 034026 (2006); C. H. Chen and C. Q. Geng, Phys. Rev. D 75, 054010 (2007).

[31] BaBar Collaboration, B. Aubert et al., arXiv:0907.1776 [hep-ex]

[32] M. Bona et al., arXiv:0709.0451 [hep-ex]

[33] S. Hashimoto et al., KEK-REPORT-2004-4 